\title{
Cambios morfológicos en gametos del barbo tigre Puntius tetrazona (Cypriniformes: Cyprinidae) e implementación de la fertilización in vitro
}

\author{
Omar Domínguez-Castanedo ${ }^{1}$, Ángel Toledano-Olivares ${ }^{2}$, David Martínez-Espinosa ${ }^{3}$ \& \\ Alejandro Ávalos-Rodríguez ${ }^{2}$ \\ 1. Programa de Doctorado en Ciencias Biológicas y de la Salud. \\ 2. Laboratorio de Bioquímica de la Reproducción, Depto. Producción Agrícola y Animal. \\ 3. Laboratorio de Sistemas Acuícolas, Depto. El Hombre y su Ambiente. Universidad Autónoma Metropolitana, unidad \\ Xochimilco. Calzada del Hueso No. 1100, Col. Villa Quietud, 04906, Coyoacán, México, D.F.; dominguezcastane- \\ do@gmail.com, legna_005@hotmail.com,maed4024@correo.xoc.uam.mx, avalosr@correo.xoc.uam.mx
}

Recibido 13-I-2014. Corregido 10-V-2014. Aceptado 12-VI-2014.

\begin{abstract}
Morphological changes in gametes of tiger barb Puntius tetrazona (Cypriniformes: Cyprinidae) and the implementation of in vitro fertilization. The production of ornamental fishes represents an economic activity of a growing number of Mexican families. Nevertheless, the reproduction of fish in captivity is one of the complications faced by farmers. This study was set up to: (i) evaluate the morphological and functional changes induced by hydration in the gametes of fish tiger barb (Puntius tetrazona; 240 samples) at tree times after hydration $(10,20$ and 30s) with classic spermograms (volume, sperm concentration, viability, motility, and normal morphology); and (ii) evaluate the implementation of in vitro fertilization based on the ovulation rate, the percentage of fertilization and hatching, and the larval numbers obtained after 72 hours. The average volume of milt was $3.0 \pm 0.7 \mu \mathrm{L}$, and the minimum, maximum and average concentration of sperm was $44.4 \times 10^{6}$ $\mathrm{spz} / \mathrm{mL}, 52.3 \times 10^{6} \mathrm{spz} / \mathrm{mL}$, and $48.1 \pm 5.9 \times 10^{6} \mathrm{spz} / \mathrm{mL}$, respectively. The viability and motility of the sperm was $84.6 \pm 3.2 \%$ and $81.5 \pm 2.2 \%$, respectively. The diameter of the sperm with/without water contact was $2.1 \pm 0.6 \mu \mathrm{m}$ and $3.8 \pm 1.0 \mu \mathrm{m}(\mathrm{p}<0.05)$; the largest diameter was recorded 30 seconds after the contact with water. For oocytes, the smaller and larger diameters were recorded at 10 and 30s, respectively (both with/without water contact); the oocytes diameters after 10 and 30 seconds of contact with water were 1.11 and $1.55 \mathrm{~mm}$, respectively. A higher ovulation rate was recorded using the in vitro fertilization: $250 \pm 50$ oocytes versus $28 \pm 09$ oocytes (during natural fertilization; $\mathrm{p}<0.05)$. Nevertheless, fertilization and hatching rates were higher for the natural fertilization ( 80 and $60 \%$, respectively). Considering the number of larvae obtained after 72 hours, our results showed a higher value for the in vitro fertilization ( $75 \pm 18$ compared to $13.4 \pm 12$ of the natural fertilization; $p<0.05)$. We propose this fish as a model for other ornamental fishes of commercial interest. Our results demonstrate that the in vitro fertilization is a very high viable option to optimize and maximize resources; besides, the reproduction management optimization under controlled conditions may enhance wild fish stocks preservation. Rev. Biol. Trop. 62 (4): 1353-1363. Epub 2014 December 01.
\end{abstract}

Key words: tiger barb, oocytes, sperm, in vitro fertilization, gamete quality, Ciprinidae, ornamental aquaculture.

La acuicultura ornamental constituye el sustento económico, de un número progresivo de familias mexicanas. Sin embargo, la reproducción de los peces en cautiverio es una de las mayores limitantes que enfrentan los productores a lo largo de este proceso productivo (Domínguez-Castanedo, 2012; MartínezEspinosa, Sánchez-Robles, Matus-Parada \&
Binqüist-Cervantes, 2013). Lamentablemente, esta situación limita el desarrollo económico del sector al no poder competir con la oferta del mercado extranjero (Donaldson, 1996; Martínez et al., 2009; Mylonas, Fostier, \& Zanuy, 2010).

El éxito de la piscicultura ornamental depende de la producción de peces con mayor 
demanda comercial, que por lo general son nativas de las regiones tropicales de América, Asia y África (Martínez et al., 2009). Así, las especies de peces que son llevadas a otros países para su comercialización, presentan cambios funcionales que derivan en diversas dificultades para su reproducción (Zohar \& Mylonas, 2001), lo que impide a la industria acuícola local competir con la internacional. Lo anterior resulta, por un lado, en la descapitalización de los productores locales y por otro, en un creciente impacto negativo en la conservación de las poblaciones silvestres de peces -ya que un gran número de especies comerciales son extraídas de sus hábitats de origen- con el aumento de la tasa de importaciones del extranjero; se calcula que hasta el $80 \%$ de los peces disponibles en el mercado mexicano son importados (Dos Anjos, Siquiera \& Amorim, 2007; Martínez et al., 2009).

La dificultad para lograr la reproducción de los peces importados se debe principalmente a las disfunciones reproductoras ocasionadas por el cambio en las condiciones de su hábitat $y$, particularmente, al desconocimiento de las características y calidad de sus gametos (Zohar \& Mylonas, 2001; Bobe \& Labbé, 2010). Los gametos de los teleósteos poseen características particulares como la presencia de un micrópilo en los ovocitos, la carencia de acrosoma en los espermatozoides y la activación de ambos gametos cuando entran en contacto con el agua (Rurangwa, Kime, Ollevier, \& Nash, 2004; Tabares, Tarazona, \& Olivera, 2005). En un tiempo relativamente breve, dependiendo de la especie, debe ocurrir la fertilización, de lo contrario pueden sobrevenir cambios morfológicos que disminuyen la viabilidad de los gametos (Vladic, Afzelius, \& Bronnikov, 2002). En concomitancia con lo anterior, su calidad depende de complejas interacciones entre factores genéticos, fisiológicos y ambientales. Estos últimos, se vuelven determinantes en la acuicultura, donde el manejo alimenticio, hacinamiento, fotoperiodo, calidad del agua e interacción con productos químicos, entre otros, se desempeñan como factores agregados de alto impacto que llegan a definir el éxito de los procesos reproductores de los teleósteos (Blaxter, 1969; Rurangwa et al., 2004).

El estudio de los gametos se centra en su mayoría, en el análisis cualitativo de los ovocitos, pues es fundamental determinar el estado de madurez en que se encuentran para llevar a cabo la fertilización. Sin embargo, actualmente se sabe sobre la importancia que tiene la calidad espermática, no sólo por su contribución genética y activación de los ovocitos, sino también, para la producción de embriones y larvas viables, mediante la integridad y calidad de su ADN (Coward, Bromage, Hibbitt, \& Parrington, 2002; Rurangwa et al., 2004). Así, la comprensión de la biología de los gametos y los factores que afectan su calidad, es el camino para perfeccionar los métodos y las técnicas necesarios para diseñar una fertilización in vitro adecuada para cada especie (Billard, Cosson, Perchec, \& Linhart, 1995b; Coward et al., 2002).

El barbo tigre Puntius tetrazona, es un pez tropical nativo de la península Malaya, y las islas de Sumatra (Indonesia) y Borneo (Malasia), así como algunas zonas de Tailandia. Es una especie ornamental de gran importancia económica a nivel internacional (Dawes, 2005; Martínez et al., 2009). Es un teleósteo de reproducción constante y muy prolífico, ya que presenta múltiples desoves durante el periodo reproductor ocurrido entre primavera y verano en su hábitat de origen. En cautividad, se ha documentado su reproducción con una frecuencia quincenal a partir de los tres meses de vida, con una producción promedio de hasta 500 ovocitos (Munro, Li-Lian, \& Nqankee, 1990). Se ha observado que cuando se extrae de sus condiciones naturales, P. tetrazona presenta algunas disfunciones reproductoras, como la interrupción súbita del comportamiento reproductor e inhibición del desove (Dawes, 2005).

Por lo anterior, es importante la generación de alternativas que permitan ampliar el conocimiento de la biología reproductora de $P$. tetrazona, a partir del estudio de sus gametos, con la finalidad de adaptar técnicas frecuentemente utilizadas en la industria de peces comestibles (los cuales se caracterizan por presentar un 
tamaño grande que facilita su manejo), a las características de los peces ornamentales. Con base en lo anterior, los objetivos del presente estudio fueron conocer las características de la calidad seminal y de los ovocitos del barbo tigre $P$. tetrazona así como los cambios morfológicos a partir de su contacto con el agua; con el propósito de evaluar e implementar la fertilización in vitro.

\section{MATERIALES Y MÉTODOS}

Este estudio se llevó a cabo en las instalaciones del Laboratorio de Sistemas Acuícolas de la Universidad Autónoma Metropolitana, unidad Xochimilco. Se utilizaron 120 parejas de la especie Puntius tetrazona, de cuatro meses de edad, sexualmente maduros y de primera reproducción adquiridos con un proveedor local, con las siguientes características morfométricas: longitud patrón: $3.25 \pm 07 \mathrm{~cm}$; altura máxima: $2.20 \pm 02 \mathrm{~cm}$ y peso: $3.07 \pm 03 \mathrm{~g}$. Los peces fueron alojados en un acuario de 80L con las siguientes condiciones controladas: $26 \pm 1{ }^{\circ} \mathrm{C}$ con un termostato de $100 \mathrm{w}$ Elite ${ }^{\circledR}$ y con filtración mecánica, biológica y química con filtros de caja Azul ${ }^{\circledR}$. El fotoperiodo se estableció de 14 horas luz por 10 horas de obscuridad, de acuerdo a los parámetros óptimos de la especie sugeridos por Munro et al. (1990). Se alimentó a los peces tres veces al día, $a d$ libitum con el alimento comercial Tetracolor ${ }^{\circledR}$ en pellets de acuerdo a Dawes (2005).

Todos los peces fueron inducidos a la reproducción de forma natural en tres ocasiones, espaciadas con 30 días de descanso cada una. En la primera inducción a la reproducción se caracterizó la calidad de los gametos en seco. En la segunda se registraron sus cambios morfológicos con diferentes tiempos posthidratación con agua. Finalmente, en la tercera ocasión, se implementó y evaluó la técnica de fertilización in vitro.

Inducción natural a la reproducción: Doce horas antes de las evaluaciones, se inició la inducción natural del desove. Para ello, se cambió 50\% del volumen de acuario con agua declorada a $26^{\circ} \mathrm{C}, 80 \mathrm{mg} / \mathrm{L}$ de dureza general y $\mathrm{pH}$ de 7.9. Para simular la vegetación se colocó el sustrato para desove, consistente en múltiples hilos tejidos de color verde (El Gato ${ }^{\circledR}$, $20 \mathrm{~cm}$ de largo, de la superficie al fondo) de acuerdo a lo sugerido por Lambert (2001). La proporción sexual utilizada fue de 1:1.

Colecta de gametos: $\mathrm{Al}$ identificar el inicio del comportamiento reproductor (momento en que la hembra fue receptiva al macho), se procedió a separar los peces de acuerdo a su sexo. Después se comprobó la ovulación y espermiación en hembras y machos respectivamente, con una ligera presión abdominal, confirmando la salida libre de los gametos por el poro genital. La colecta de las muestras fue de acuerdo a Yamamoto (1961) y Rodríguez (1992), con las siguientes variaciones: se secó el poro genital con un paño limpio y se realizó un ligero masaje abdominal de dirección opercular - caudal a partir del inicio de la aleta dorsal. El semen se colectó en tubos capilares sin heparina de $75 \mathrm{~mm}$ Propper ${ }^{\circledR}$ (uno por individuo) y los ovocitos en un vidrio de reloj de $5 \mathrm{~cm}$ de diámetro Pyrex ${ }^{\circledR}$ para cada hembra. Este procedimiento se mantuvo en las etapas de la investigación en que se colectaron los gametos.

Caracterización de la calidad de los gametos en seco: Los gametos masculinos se analizaron con espermogramas clásicos. $\mathrm{Se}$ evaluó el volumen, la concentración, motilidad, morfología normal y viabilidad de acuerdo a Rodríguez (1992) en cada uno de los sujetos de estudio. En el caso de las hembras, se obtuvo la tasa de ovulación y el diámetro de los ovocitos de acuerdo a Lahnsteiner, Soares, Ribeiro y Dinis (2009).

Evaluación del cambio en la morfología de los gametos pos-hidratación: Para registrar el cambio en el diámetro de los ovocitos y la cabeza de los espermatozoides, éstos fueron fotografiados y posteriormente, medidos en cuatro momentos. El primer momento fue en seco; posteriormente, a esta lectura fueron agregados $200 \mu \mathrm{L}$ de agua a los gametos. Las 
siguientes mediciones se realizaron a los 10 , 20 y 30 segundos. Para evaluar la motilidad, se observó la cantidad de espermatozoides móviles posterior a la hidratación a los 10,30 y 60 segundos, de acuerdo a Rodríguez (1992).

El tiempo de motilidad de los espermatozoides, se registró a partir de su activación y hasta la pérdida de la misma en las muestras de todos los individuos. Para la observación y medición de los gametos se utilizó un microscopio Nikon eclipse $80 i^{\circledR}$ y el programa ImagePro Plus $5.1^{\circledR}$.

Evaluación de las técnicas de fertilización: Se evaluaron a partir de dos grupos experimentales (reproducción in vitro y natural) por triplicado. Fueron empleadas 20 parejas de $P$. tetrazona en cada réplica de los grupos experimentales (60 parejas por grupo). Los peces de cada réplica se colocaron en acuarios de $20 \mathrm{~L}$ a $26 \pm 1^{\circ} \mathrm{C}$ para la inducción natural a la reproducción, tal como se mencionó anteriormente. Para la evaluación de ambas técnicas de fertilización se registraron los siguientes indicadores: la tasa de ovulación (número total de ovocitos obtenidos por hembra), el porcentaje de fertilización (número de ovocitos fertilizados a las $4 \mathrm{~h}$ ), el porcentaje de eclosión y número de larvas obtenidas a las 72 horas.

Grupo de fertilización in vitro: Una vez observado el inicio del comportamiento reproductor de los peces de este grupo, se palpó el abdomen de todas las hembras para comprobar la ovulación y en su caso, estimular la salida de ovocitos por el poro genital, antes de su desove natural. En las hembras en las que no se registró, fueron regresadas al acuario de donde fueron extraídas y se repitió la acción 30 minutos después. Una vez obtenidos los ovocitos, se evaluó la técnica con los indicadores descritos previamente. Se colectó el semen en tubos capilares y se depositó sobre los ovocitos en el vidrio de reloj (la totalidad de los gametos colectados por cada pareja). Inmediatamente se agregaron $10 \mathrm{~mL}$ de agua $\left(26^{\circ} \mathrm{C} ; \mathrm{pH}\right.$ de $7.0 ; 80 \mathrm{mg} / \mathrm{L}$ de dureza general) y se mezclaron los gametos por pareja, con movimientos circulares del vidrio de reloj durante 60 segundos para permitir la fertilización.

Grupo de fertilización natural: Al finalizar el comportamiento reproductor y el desove, se retiraron los peces del acuario y se realizó la evaluación de los indicadores antes mencionados, y se corroboró el desove de todas las hembras mediante la dilatación de su poro genital y la salida de ovocitos ovulados remanentes en el lumen del ovario con un ligero masaje. La evaluación de los indicadores se realizó mediante la sumatoria de los embriones obtenidos en cada acuario (réplica) dividida entre el total de hembras presentes en cada uno (20 parejas), debido a que se permitió el desove de forma natural a los peces de cada acuario sobre el sustrato de desove, contrario al procedimiento realizado para la fertilización in vitro.

Para determinar la existencia de diferencias significativas entre los tiempos de hidratación de los gametos se realizaron análisis de varianza de una vía (ANOVA). De existir éstas, se procedió a una comparación de medias múltiples por la prueba de Tukey. Para evaluar las diferencias entre los indicadores de las técnicas de fertilización se realizaron pruebas de t-student. El nivel de significancia en las pruebas fue de $p<0.05$.

\section{RESULTADOS}

Caracterización de la calidad de los gametos: Los valores promedio del análisis de los gametos se muestran en el cuadro 1. El volumen de semen de los eyaculados fluctuó entre 2 y $5 \mu \mathrm{L}$ con $3 \pm 0.7 \mu \mathrm{L}$ en promedio. La concentración espermática mínima y máxima fue de $44.48 \times 10^{6}$ y $52.3 \times 10^{6}$ espermatozoides $(\mathrm{spz}) / \mathrm{mL}$, respectivamente. En promedio se registraron $48.1 \pm 5.9 \times 10^{6} \mathrm{spz} / \mathrm{mL}$. El porcentaje de viabilidad en los espermatozoides se registró entre 78.6 y $88.8 \%$ con promedio de $84.68 \pm 3.27 \%$. La figura 1 muestra un espermatozoide vivo y otro muerto, por la pérdida de permeabilidad en la membrana, durante esta prueba. La prueba de motilidad registró un mínimo y máximo de 78.4 y $88.4 \%$ de 
CUADRO 1

Caracterización de los gametos sin contacto con el agua en P. tetrazona

TABLE 1

Characterization of gametes without contact with water in P. tetrazona

\begin{tabular}{llc} 
Gameto & \multicolumn{1}{c}{ Prueba } & Resultados \\
Espermatozoides & Volumen $(\mu \mathrm{L})$ & $3.0 \pm 0.7$ \\
& Concentración espermática $(\mathrm{spz} / \mathrm{mL})$ & $48.10 \pm 5.9 \times 10^{6}$ \\
& Viabilidad $(\%)$ & $84.68 \pm 3.27$ \\
& Motilidad progresiva (\%) & $81.53 \pm 2.28$ \\
Ovocitos & Morfología normal (\%) & $85.27 \pm 6.7$ \\
& Tasa ovulatoria (no. de ovocitos) & $260 \pm 110$ \\
& Diámetro $(\mathrm{mm})$ & $1.0 \pm 0.03$ \\
\hline
\end{tabular}

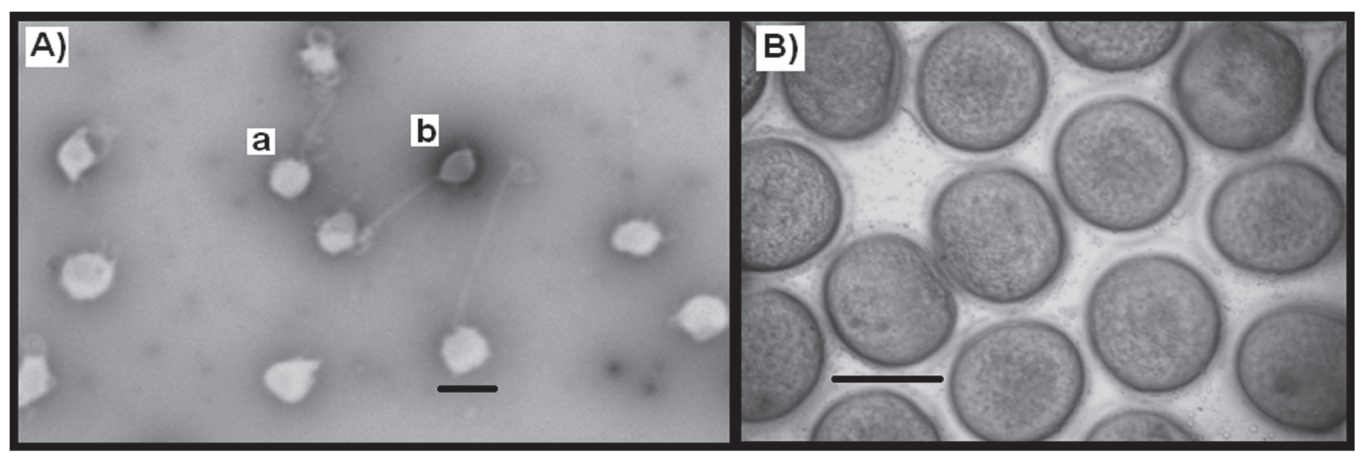

Fig. 1. A) Espermatozoides de P. tetrazona a) vivo b) espermatozoide muerto en la prueba de viabilidad por la tinción con eosina-nigrosina, barra $=2 \mu \mathrm{M}$. B) Ovocitos durante el conteo de la tasa ovulatoria, barra $=1 \mathrm{~mm}$.

Fig. 1. A) Sperm of $P$. tetrazona a) live b) death sperm during the viability test by staining of eosin-nigrosin, bar=2 $\mu \mathrm{M}$. B) Oocytes durng ovulatory rate count, bar $=1 \mathrm{~mm}$.

espermatozoides móviles respectivamente, con promedio de $81.53 \pm 2.28 \%$. En las hembras, la tasa ovulatoria registrada fue de $260 \pm 110$ ovocitos en promedio, con diámetro de de $1.0 \pm 0.03 \mathrm{~mm}$.

Caracterización de los cambios morfológicos a través del tiempo: Semen. El diámetro de los espermatozoides fluctuó de $2.16 \pm 0.2$ a $2.79 \pm 0.3 \mu \mathrm{m}$ y $3.84 \pm 0.3$ a $4.86 \pm 0.31 \mu \mathrm{m} \sin$ contacto y en contacto con el agua respectivamente. Se registró el menor valor en el diámetro de los espermatozoides a los diez segundos sin estar en contacto con el agua. El diámetro mayor se registró a los 30 segundos, estando en contacto con el agua (Fig. 2). En los espermatozoides sin contacto con el agua no se registraron diferencias entre los diferentes tiempos. En los espermatozoides con contacto con el agua, se detectaron diferencias estadísticas sólo entre el grupo de 30 segundos con respecto a los demás (Tukey, $\mathrm{p}<0.05$ ). Finalmente, se registraron diferencias estadísticas entre los tres tiempos del grupo que no estuvo en contacto con el agua, con respecto al grupo en contacto con el agua (Tukey, $\mathrm{p}<0.05$ ).

Ovocitos. Los ovocitos de menor diámetro se registraron a los diez segundos sin contacto con el agua. El diámetro mayor del grupo de ovocitos en contacto con el agua se registró a los 30 segundos. Los diámetros de los ovocitos en contacto con el agua fluctuaron entre $1.11 \mathrm{y}$ $1.55 \mathrm{~mm}$ a los diez y los 30 segundos, respectivamente (Fig. 3). En el grupo de los ovocitos 


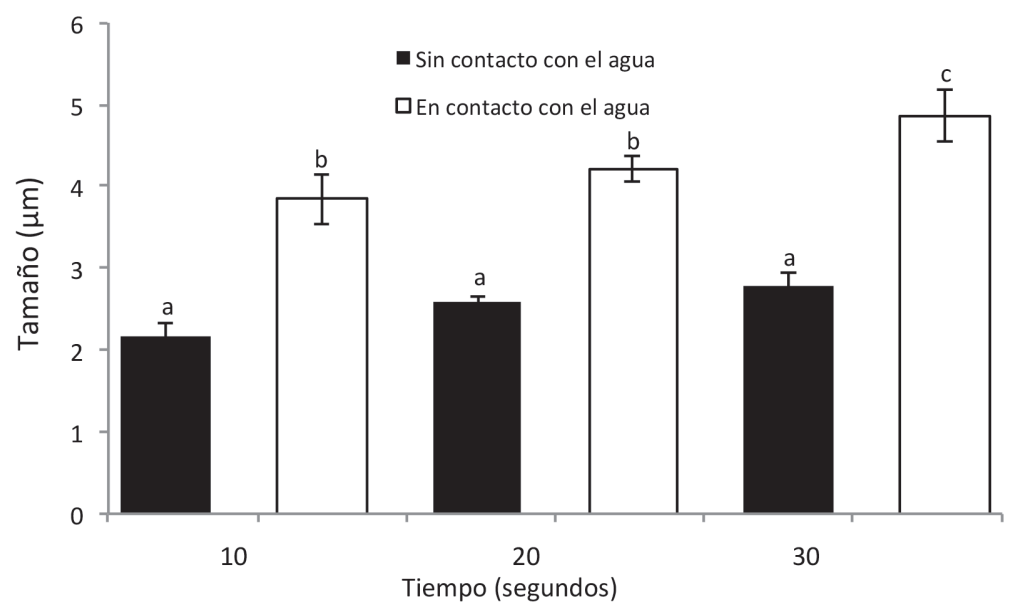

Fig. 2. Diámetro de los espermatozoides a diferentes tiempos de hidratación.

Fig. 2. Diameter of spermatozoa at different times of hydration.

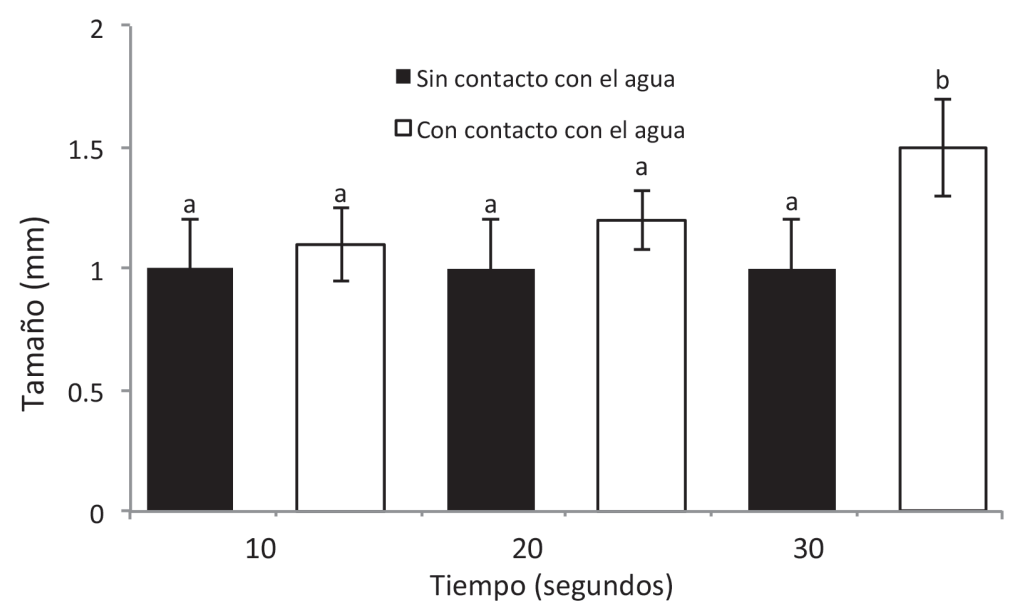

Fig. 3. Diámetro de los ovocitos a diferentes tiempos de hidratación.

Fig. 3. Diameter of oocytes at different times of hydration.

en seco no se observaron cambios en el diámetro $(1.0 \mathrm{~mm})$ en ninguno de los tiempos. Se registraron diferencias estadísticas significativas (Tukey, $\mathrm{p}<0.05$ ) entre los ovocitos hidratados a los 30 segundos con respecto a todos los demás tiempos de ambos grupos.

Caracterización de los cambios en la motilidad de los espermatozoides: El porcentaje registrado de espermatozoides móviles a partir de su activación fue de $81.53 \pm 2.28 \%$. La motilidad a los 30 segundos se redujo a $63 \%$ de espermatozoides. A los 60 segundos se registró el $100 \%$ de espermatozoides sin motilidad.

Fertilización in vitro: Tasa de ovulación. La técnica de fertilización que registró mayor tasa de ovulación fue in vitro con $250 \pm 50$ ovocitos frente a $28 \pm 09$ ovocitos ovulados en la técnica natural. Se detectaron diferencias 


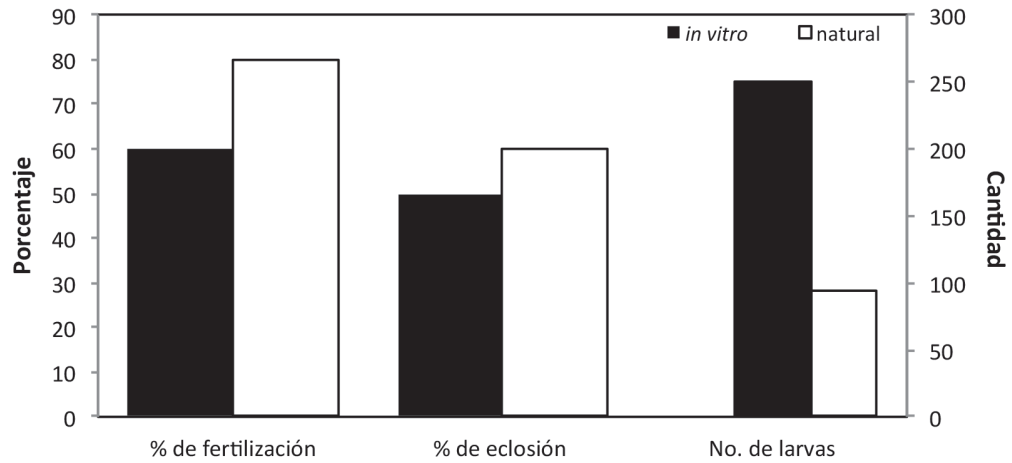

Fig. 4. Porcentajes de fertilización, eclosión y número de larvas de las técnicas de fertilización in vitro y natural en $P$. tetrazona.

Fig. 4. Percentages of fertilization, hatching and number of larvae of in vitro and natural techniques of fertilization in $P$. tetrazona.

estadísticas significativas entre las dos técnicas de fertilización evaluadas para este indicador (t-student, $\mathrm{p}<0.05$ ).

Porcentaje de fertilización. Contrario a lo registrado en el indicador anterior, el porcentaje de fertilización más elevado se observó en la técnica de fertilización natural, con $80 \%$ de los ovocitos fertilizados, frente al $60 \%$ registrado en la técnica in vitro (Fig. 4). Se detectaron diferencias estadísticas significativas en este indicador de evaluación de las técnicas de reproducción ( $\mathrm{t}$-student, $\mathrm{p}<0.05$ ).

Porcentaje de eclosión. El porcentaje registrado de embriones eclosionados fue mayor en la técnica de fertilización natural con $60 \%$ de larvas, con respecto al 50\% registrado en el grupo de la fertilización in vitro. No se detectaron diferencias estadísticas significativas (Fig. 4).

Número total de larvas obtenidas a las 72 horas. El número mayor de larvas obtenidas fue en la fertilización in vitro con $75 \pm 18$, en contraste con $13.44 \pm 12$ registradas en la técnica de fertilización natural. Se registraron diferencias estadísticas significativas entre las técnicas de fertilización (t-student, $\mathrm{p}<0.05$ ).

\section{DISCUSIÓN}

El estudio de la biología reproductora de los peces de importancia económica, es la base para lograr que la acuicultura con fines de consumo u ornamentales, aumente su rentabilidad, logrando con ello perpetuarla a través del tiempo. El estudio de los gametos (a partir de cada uno de sus indicadores de calidad) es la clave para el diseño e implementación de técnicas y tecnologías que permitan intensificar la producción (Coward et al., 2002; Hajirezaee, Amiri, \& Mirvaghefi, 2010).

La morfología anormal de los espermatozoides es un indicador de la reducción en su capacidad fertilizante por sus deficiencias funcionales y la reducción de su motilidad. Es conocido que en las muestras seminales se encuentra un elevado porcentaje de anormalidades morfológicas, usualmente compensadas por las grandes concentraciones existentes de gametos totales (Van Look \& Kime, 2003; Rurangwa et al., 2004; Lavara, Mocé, Lavara, Viudes de Castro, \& Vicente, 2005). La morfología normal de los espermatozoides de P. tetrazona es descrita por Ohta y Matsuda (1995), como células de $28.9 \mu \mathrm{m}$ de longitud compuestas por una cabeza esférica de $1.47 \mu \mathrm{m}$ sin acrosoma, una pieza media y un flagelo. El diámetro de la cabeza de los espermatozoides que se registró en el presente trabajo fue menor a lo reportado por estos autores, lo que pudo deberse a que su investigación fue realizada a través de microscopia electrónica, obteniendo mayor exactitud en sus mediciones. 
Uno de los indicadores más utilizados para la evaluación de la calidad seminal en los teleósteos es la concentración de espermatozoides en el líquido seminal (Rurangwa et al., 2004). No obstante, no hay una unificación de criterios para el reporte de los resultados. Rurangwa et al. (2004) sugirió que la manera más adecuada de reportar este indicador es el número de espermatozoides presentes en un $\mathrm{mL}$ de semen. Con ello, se permite comparar los resultados independientemente del peso de los peces, como se reporta frecuentemente.

La concentración espermática de $P$. tetrazona es más baja que las reportadas en los ciprínidos Barbus aeneus con $13.75 \times 10^{6} \mathrm{~mm}^{3}$ (Vlok \& Van Vuren, 1988); Cyprinus carpio con $24.70 \times 10^{9} \mathrm{~mm}^{3}$ (Clemens \& Grant, 1985), $28.0 \times 10^{9} \mathrm{~mm}^{3}$ (Musselius, 1951), y $6.52 \times 10^{6}$ $\mathrm{mm}^{3}$ (Kruger, Smit, Van Vuren, \& Ferreira, 1984) y Ctenopharyngodon idella con $9.37 \times 10^{9}$ en su grupo control (Metwally \& Fouad, 2009). Xiao, Zhang, Hu, y Xu (2006), reportaron en el barbo rosy, $P$. conchonius - especie ornamental con características similares a las del barbo tigre - una concentración significativamente más elevada $\left(3.1 \times 10^{11} \mathrm{spz} / \mathrm{mL}\right)$ que las observadas en el presente estudio para $P$. tetrazona.

Los espermatozoides de los teleósteos, permanecen inmóviles durante su almacenamiento en los conductos deferentes y la eyaculación. La motilidad y activación metabólica se adquiere al iniciar el contacto con el agua o fluidos ováricos, perdiéndose pocos segundos después (Billard, Cosson, Crim, \& Suquet 1995a; Bromage \& Roberts, 1995; Kime et al., 2001; Linhart, Cosson, Mims, Shelton, \& Rodina 2002; Rurangwa et al., 2004).

El patrón de motilidad espermática en $P$. tetrazona obtenido en este trabajo fue similar al reportado en otros teleósteos ovíparos como Cyprinus carpio (Billard et al., 1995b), donde inició simultáneamente en todos los espermatozoides y se mantuvo durante $30-40 \mathrm{~s}$. Al entrar en contacto con el agua, cambió su morfología debido a la presión osmótica y perdieron la motilidad entre los 60 y 90s. Estos resultados son equiparables a lo reportado en la presente investigación, y muy inferiores a los resultados reportados para el grupo control de Xiao et al. (2006) en P. conchonius, donde la motilidad se prolongó hasta por $30 \mathrm{~min}$ en el $80 \%$ de los espermatozoides.

Metwally \& Fouad (2009) reportaron en Ctenopharyngodon idella una motilidad del $83.2 \%$ de los espermatozoides durante $36.4 \mathrm{~s}$ en su grupo control, a este mismo tiempo se observó en $P$. tetrazona, una disminución mayor de la motilidad. Estas diferencias pueden ser explicadas por la utilización de soluciones fisiológica para peces y buffer de sodio hipoosmótico durante la evaluación espermática en otros estudios. El decremento en la motilidad en tan corto tiempo se explica por la disminución en el contenido de ATP intracelular, ya que al término de la fase de motilidad, es hidrolizado entre 50 y $80 \%$ de éste (Billard et al., 1995b; Coward et al., 2002). La activación de la motilidad en los peces de la familia Cyprinidae, está regulada por el cambio en la presión osmótica entre el plasma seminal y el agua (Billard et al., 1995b). La activación es inducida por la exposición a un medio hipotónico y la duración en el periodo de motilidad está dada por el grado de diferencia entre las condiciones ambientales (hostiles para el espermatozoide) y las características del fluido seminal (Bromage \& Roberts, 1995; Takai \& Morisawa, 1995; Coward et al., 2002; Rurangwa et al., 2004; Hoang et al., 2010). Asimismo, cuando el espermatozoide es activado, sufre cambios morfológicos al hidratarse, entre los que se incluyen el estiramiento de la membrana provocado por el aumento en el volumen de líquido intracelular debido a la pérdida de polaridad, ocasionada por las diferencias de presión osmótica (Tabares et al., 2005) y el aumento en el diámetro de los espermatozoides, como se documentó en la presente investigación.

Por otro lado, de acuerdo a Rurangwa et al. (2004), la viabilidad de los espermatozoides se define como su capacidad para moverse y fertilizar un ovocito. En el presente estudio se evaluó la viabilidad en función de la integridad de su membrana, los resultados son similares a los mínimos registrados en Barbus aeneus (Vlok \& Van Vuren, 1988). 
Por su parte, la morfometría de los ovocitos es utilizada frecuentemente como un parámetro para evaluar su calidad, debido a que provee un estimado del aporte de los padres a la descendencia. Los ovocitos más grandes poseen usualmente mayores cantidades de vitelo, lo que brinda mayores posibilidades de sobrevivir a las larvas (Springate \& Bromage, 1985). El número y tamaño de los ovocitos es afectado por diversos factores, como la condición nutricional de la madre, su edad y tamaño (Blaxter, 1969), lo que podría explicar el número reducido de ovocitos ovulados por las hembras de la presente investigación, ya que fueron jóvenes de primera reproducción. El diámetro de los ovocitos fue similar al reportado por Petrovický (2002) en esta especie.

Los ovocitos son activados por el agua cuando son liberados en soluciones hipotónicas. La membrana vitelina se torna opaca y ocurren cambios en su permeabilidad. Si los espermatozoides no fertilizan al ovocito inmediatamente, su tasa de viabilidad se reduce significativamente en un tiempo muy corto (Coward et al., 2002). Cuando los ovocitos son liberados en el agua se hidratan, lo que explica el aumento observado en su diámetro en el presente trabajo, lo que produce que el micrópilo se cierre, y se evite la fertilización (Tabares et al., 2005).

Dado el cambio morfológico registrado en los gametos cuando entran en contacto con el agua y las implicaciones con su viabilidad, se sugiere realizar la fertilización in vitro en $P$. tetrazona, durante los primeros diez segundos posteriores a su activación o "en seco" (Yamamoto, 1961; Bromage \& Roberts, 1995). Esto asegura que la activación de las células sexuales de ambos sexos ocurra hasta que se encuentren en contacto. Lo anterior permite aumentar el tiempo de viabilidad y por ende, el porcentaje de fertilización.

La técnica de fertilización in vitro fue más eficiente que la técnica natural, debido a que se logró obtener mayor cantidad de larvas. Lo anterior, a pesar de registrar los porcentajes menores de fertilización y de eclosión. El éxito de la reproducción artificial radicó en la posibilidad de extraer la totalidad de los ovocitos ovulados en contraste con la técnica natural, donde los peces desovaron sólo una pequeña cantidad, manteniendo el resto de los ovocitos ovulados en el interior del lumen del ovario.

Lo anterior demuestra la viabilidad en la implementación de la fertilización in vitro en peces ornamentales por las ventajas que ésta proporcionó. Además, permite evaluar a los machos reproductores para seleccionar aquellos que posean características genéticas deseables. Esta técnica también reduce el número de machos y hembras utilizados en la producción, y por ende, abate los costos de manutención en los reproductores. También favorece la realización de la criopreservación del semen y la creación de bancos de gametos con los peces seleccionados (Yamamoto, 1961; Beirão Soares, Herráez, Dinis, \& Cabrita, 2009; Hajirezaee et al., 2010).

Con el presente estudio, se contribuye al conocimiento de la biología reproductora del pez tropical P. tetrazona y se propone la utilización de la fertilización in vitro, como modelo para otros peces ornamentales. La implementación de técnicas utilizadas comúnmente en peces comestibles - caracterizados por tener un tamaño fácilmente manejable - en una industria en pleno crecimiento con grandes problemáticas, es una manera de crear alternativas con el fin de optimizar y maximizar sus recursos, preservando las poblaciones naturales de peces.

\section{AGRADECIMIENTOS}

A Liliana García-Calva por sus sugerencias para mejorar el manuscrito y Bruno A. Marichal-Cancino por la revisión del manuscrito y la elaboración del abstract. A Jorge A. González-Santos por la edición de las figuras. A los revisores anónimos por sus comentarios y sugerencias.

\section{RESUMEN}

El objetivo del presente estudio fue conocer las características de los gametos de Puntius tetrazona $(\mathrm{n}=240)$, los 
cambios morfológicos a partir de su activación mediante espermogramas cásicos y por otro lado, se evaluó la implementación de la fertilización in vitro a partir de la tasa ovulatoria, el \% de fertilización y eclosión y el número de larvas vivas a las $72 \mathrm{~h}$. El volumen promedio de semen fue de $3.0 \pm 0.7 \mu \mathrm{L}$. La concentración espermática mínima, máxima y promedio fue $44.48 \times 10^{6} \mathrm{spz} / \mathrm{mL}, 52.3 \times 10^{6} \mathrm{spz} /$ $\mathrm{mL}$ y $48.1 \pm 5.9 \times 10^{6} \mathrm{spz} / \mathrm{mL}$, respectivamente. La viabilidad promedio fue de $84.68 \pm 3.27 \%$. La motilidad promedio fue $81.53 \pm 2.28 \%$. El diámetro de los espermatozoides fluctuó entre $2.16 \pm 0.2$ y $2.79 \pm 0.3 \mu \mathrm{m} ; 3.84 \pm 0.3$ y $4.86 \pm 0.31 \mu \mathrm{m}$ sin y con contacto con el agua respectivamente, con diferencias significativas. El diámetro mayor fue a los 30 s en contacto con el agua. Los ovocitos de menor y mayor diámetro se registraron a los diez y 30 s sin y con contacto con el agua respectivamente. Los diámetros de los ovocitos en diez y 30 s en contacto con el agua fluctuaron entre 1.11 y $1.55 \mathrm{~mm}$ respectivamente. La mayor tasa ovulatoria fue en la fertilización in vitro con $250 \pm 50$ ovocitos frente a $28 \pm 09$ de la natural, con diferencias significativas. Los porcentajes de fertilización y eclosión fueron más elevados en la fertilización natural con $80 \%$ y $60 \%$ respectivamente. Se registraron $75 \pm 18$ larvas a las 72 horas en el grupo in vitro comparado con $13.4 \pm 12$ larvas de la fertilización natural. Con lo anterior, la técnica que permitió mayor cantidad de larvas fue la de fertilización in vitro.

Palabras clave: barbo tigre, ovocitos, espermatozoides, fertilización in vitro, calidad de gametos, Ciprinidae, acuacultura ornamental.

\section{REFERENCIAS}

Beirão, J., Soares, F., Herráez, M. P., Dinis, M. T., \& Cabrita, E. (2009). Sperm quality evaluation in Solea senegalensis during the reproductive season at cellular level. Theriogenology, 72, 1251-1261.

Billard, R., Cosson, J., Crim, L. W., \& Suquet, M. (1995a). Sperm physiology and quality. In N. Bromage \& R. Roberts (Eds.), Broodstock management and egg and larval quality (pp. 25-52). Oxford, Reino Unido: Blackwell.

Billard, R., Cosson, G., Perchec, G., \& Linhart, O. (1995b). Biology of sperm and artificial reproduction in carp. Aquaculture, 129, 95-112.

Blaxter, J. H. (1969). Development: eggs and larvae. In W. S. Hoar, \& D. J. Randal (Eds.), Fish Physiology (pp. 177). New York, US: Academic Press.

Bobe, J. \& Labbé, C. (2010). Egg and sperm quality in fish. General and Comparative Endocrinology, 165, 535-548.

Bromage, N. \& Roberts, J. R. (1995). Broodstock management and egg and larval quality. Oxford, Reino Unido: Blackwell Science.
Clemens, H. P. \& Grant, F. B. (1985). The seminal thinning response of carp (Cyprinus carpio) and rainbow trout (Salmo gairdnerii) after injections of pituitary extracts. Copeia, 2, 174-177.

Coward, K., Bromage, N. R., Hibbitt, O., \& Parrington, J. (2002). Gametogenesis, fertilization and egg activation in teleost fish. Reviews in Fish Biology and Fisheries, 12, 33-58.

Dawes, J. (2005). Complete encyclopedia of the freshwater aquarium. Canada and US: Firefly Book.

Domínguez-Castanedo, O. (2012). Los Sistemas Acuícolas de Recirculación: ¿una alternativa para el cultivo sustentable de peces ornamentales en el Estado de Morelos? Sociedades Rurales, Producción y Medio Ambiente, 12(24), 207-225.

Donaldson, M. E. (1996). Manipulation of reproduction in farmed fish. Animal Reproduction Science, 42, 381-392.

Dos Anjos, J. D. B., Siquiera, J. A., \& Amorim, R. M. S. (2007). Comercio de peixes ornamentais do Estado de Amazonas, Brasil. Sociedade Brasileira de Ictiologia, 87, 4-5.

Hajirezaee, S., Amiri, B. M., \& Mirvaghefi, A. (2010). Fish milt quality and major factors influencing the milt quality parameters: A review. African Journal of Biotechnology, 9(54), 9148-9154.

Hoang, M., Kyu, H., Hwa, B., Seon, M., Hyun, M., Uie, J., \& Jin, Y. (2010). Effects of varying dilutions, pH, temperature and cations on spermatozoa motility in fish Larimichthys polyactis. Journal of Environmental Biology, 32, 271-276.

Kime, D. E., Van Look, K. J. W., Mcallister, B. G., Huysekens, G., Rarangwa, E., \& Ollevier, F. (2001). Computer-assisted sperm analysis CASA as a tool for monitoring sperm quality in fish. Comparative Bioquemistry and Physiology, part C, 130, 425-433.

Kruger, J. C., Smit, G. L., Van Vuren, J. H. J., \& Ferreira. T. J. (1984). Some chemical and physical characteristics of the semen of Cyprinus carpio L. and Oreochromis mossambicus (Peters). Journal of Fish Biology, 24, 263-272.

Lahnsteiner, F., Soares, F., Ribeiro, L., \& Dinis, T. M. (2009). Egg quality determination in teleost fish. In E. Cabrita, V. Robles, \& P. Herraez (Eds.), Methods in reproductive aquaculture (pp. 149-173). US: Taylor \& Francis Group, CRC Press.

Lambert, D. 2001. A practical guide to breeding your freshwater fish. Ingland: Barron's.

Lavara, R., Mocé, E., Lavara, F., Viudes de Castro, M. P., \& Vicente, S. J. (2005). Do parameters of seminal quality correlate with the results of an on-farm inseminations rabbit? Theriogenology, 64, 1130-1141.

Linhart, O., Cosson, J., Mims, S. D., Shelton, W. I., \& Rodina, M. (2002). Effects of ions on the motility 
of fresh and demembranated paddlefish (Polyodon spathula) spermatozoa. Reproduction, 124, 713-719.

Martínez-Espinosa, D., Durán, E. E., Erna, C. A., Hernández, E. D., Galván, A., Goletto, A., Martínez, K., Malpica, S. A., Morfin, S. M., Hernández, A. J., \& Hernández, A. (2009). Plan Rector para la piscicultura de ornato del Estado de Morelos. SAGARPA.

Martínez-Espinosa, D., Sánchez-Robles, J., Matus-Parada, J., \& Binqüist-Cervantes, G. (2013). Análisis de los factores que condicionan la idoneidad de la estructura productiva de las granjas acuícolas de peces de ornato del estado de Morelos. Sociedades Rurales, Producción y Medio Ambiente, 13(25), 93-114.

Metwally, M. A. A. \& Fouad, M. I. (2009). Effects of L-Ascorbic Acid on Sperm Viability in Male Grass Carp (Ctenopharyngodon idellus). Global Veterinaria, 3(2), 132-136.

Munro, A. D., Li-Lian, C., \& Nqankee, K. (1990). Preliminary observations on environmental control of ovulation and spawning in a small tropical Cyprinid (Barbus tetrazona). Japan: Proceedings of the Second Asian Fish Forum.

Musselius, V. A. (1951). How to store carp milt and to determine its quality. Rybnoe Khozyaistvo, 27, 51-53.

Mylonas, C. C., Fostier, A., \& Zanuy, S. (2010). Broodstock management and hormonal manipulations of fish reproduction. General and Comparative Endocrinology, 165, 516-534.

Ohta, T. \& Matsuda, M. (1995). Sperm morphology and IMP distribution in membranes of spermatozoa of cyprinid fishes. Cell Structure and Function, 20, 293-300.

Petrovický, I. (2002). Peces de acuario. España: Tikal Ediciónes.

Rodríguez, G. M. (1992). Técnicas de evaluación cuantitativa de la madurez gonádica en peces. México: A.G.T. Editor, S.A.
Rurangwa, E., Kime, D. E., Ollevier, F., \& Nash, P. J. (2004). The measurement of sperm motility and factors affecting sperm quality in cultured fish. Aquaculture, 234, 1-28.

Springate, J. \& Bromage, R. N. (1985). Effect of egg size on early growth and survival in rainbow trout (Salmo gairdneri Richardson). Aquaculture, 47, 163.

Tabares, J. C., Tarazona, A. M., \& Olivera, M. A. (2005). Fisiología de la activación del espermatozoide en peces de agua dulce. Revista Colombiana de Ciencias Pecuarias, 18(2), 149-161.

Takai, H. \& Morisawa, M. (1995). Changes in intracelular $\mathrm{K}^{+}$concentrations caused by external osmolarity change regulate sperm motility of marine and freshwater teleosts. Journal of Cell Science, 108, 1175-1181.

Van Look, K. J. K. \& Kime, E. D. (2003). Automated sperm morphology analysis in fishes: the effect of mercury on goldfish sperm. Journal of Fish Biology, 63, 1020-1033.

Vladic, T. V., Afzelius, B., \& Bronnikov, E. G. (2002). Sperm quality as reflected through morphology in salmon alternative life histories. Biology of Reproduction, 66, 98-105.

Vlok, W. \& Van Vuren, J. H. J. (1988). Physical composition of the semen of Barbus aeneus, the smallmouth yellowfish (Cyprinidae). Comparative Biochemical Physiology, 90(3), 387-389.

Xiao, Q., Zhang, S., Hu, J., \& Xu, Y. (2006). Sperm of rosy barb (Puntius conchonius) as an in vitro assay system of nonylphenol cytotoxicity. Journal of Environmental Science, 18(3), 417-419.

Yamamoto, T. (1961). Physiology of fertilization in fish eggs. International Review of Cytology, 12, 361-405.

Zohar, Y. \& Mylonas, C.C. (2001). Endocrine manipulations of spawning in cultured fish: from hormones to genes. Aquaculture, 197, 99-136. 
